\title{
Uma Versão Intervalar do Método de Segmentação de Imagens Utilizando o $K$-means
}

A. TAKAHASHI, B.R.C. BEDREGAL ${ }^{2}$, Departamento de Informática e Matemática Aplicada, UFRN, Campus Universitário, Lagoa Nova, 59072-970 Natal, RN, Brasil.

A. LYRA ${ }^{3}$, Universidade Potiguar, Av. Salgado Filho 1610, Lagoa Nova, 59056-000 Natal, RN, Brasil.

\begin{abstract}
Resumo. Uma etapa importante em processamento de imagens digitais é a segmentação de imagens, pois, esta etapa é o primeiro passo no processo de análise de imagens, e, a minimização/controle de qualquer erro neste passo é fundamental para um melhor resultado da análise. Atualmente, existem diversos métodos de segmentação de imagens, dentre eles o k-means, e muitas pesquisas são realizadas visando o desenvolvimento de métodos para processamento de imagens digitais cada vez mais precisos. O uso da matemática intervalar associada ao processamento de imagens digitais tem como objetivo controlar possíveis erros computacionais. Processamento de imagens digitais intervalares é uma teoria recente, e, será apresentado neste estudo, a definição de imagens digitais intervalares juntamente com seu processamento, e nesse dentro desse processamento, a segmentação de imagens digitais intervalares, utilizando o método $k$-means intervalar, que tem como base o método de agrupamento $k$-means.
\end{abstract}

\section{Introdução}

Nos últimos anos, a segmentação de imagens vem sendo base de muitas pesquisas realizadas na área de processamento de imagens digitais, e isto se deve a sua grande importância para a análise de imagens, uma vez que, a segmentação de imagens é o primeiro passo para análise de imagens, e o resultado dessa análise é dependente de uma boa segmentação de imagens.

O processo de segmentação de imagens consiste em subdividir a imagem em várias partes ou objetos significativos [5], e este processo, bem como o seu nível de aprofundamento é dependente do problema a ser resolvido através da análise de imagens. A precisão nesta fase determina um eventual sucesso ou um fracasso no processo de análise de imagens por meio computacional, e uma atenção considerável deve ser tomada na busca de um método de segmentação de imagens eficiente.

\footnotetext{
${ }^{1}$ adriana@dimap.ufrn.br

22bedregal@dimap.ufrn.br

3 aarao.lyra@gmail.com
} 
A matemática intervalar introduzida por R. E. Moore [11, 12, 13] tem sido muito utilizada associada com diversas áreas, com o objetivo de obter um controle rigoroso de diversos tipos de erros computacionais envolvendo representações finitas de números reais [7]. Entre uma dessas áreas associadas com a matemática intervalar está o processamento de imagens digitais. Esta idéia foi considerada por Lyra $[9,10]$, onde, a representação de uma imagem digital é feita através de uma imagem digital intervalar, ou resumidamente, imagem intervalar, cujos pixels são representados por valores intervalares em vez de pontuais. A proposta de considerar imagens intervalares no processamento de imagens é obter um controle maior dos erros computacionais, tanto durante a aquisição de imagens, quanto no próprio processamento para análise de imagens.

Analogamente, o uso da matemática intervalar em métodos de segmentação de imagens tem como um dos objetivos controlar possíveis erros computacionais, tanto na compilação de algum método de segmentação, como na manipulação da imagem, dando uma maior acuracidade no resultado final do método de segmentação.

Dentre os diversos métodos para segmentação de imagens existentes, por exemplo, métodos baseados em crescimento de regiões, limiarização, divisão e fusão, agrupamento (ou clustering) $[2,4,5,6]$, entre outros, não existe um método padrão capaz de satisfazer todas as aplicações em tratamento de imagens, a escolha do melhor método deve ser avaliado de acordo com o problema a ser resolvido. O método $k$-means é um método por agrupamentos $[2,3,6,8]$, e o método $k$-means utilizado para segmentação de imagens é baseado na similaridade da intensidade luminosa das imagens. Os algoritmos para a segmentação de imagens monocromáticas seguem, geralmente, duas estratégias, baseados na descontinuidade ou na similaridade dos valores de níveis de cinza. O método de agrupamento por $k$-means é baseado na similaridade dos níveis de cinza da imagem. Neste estudo foi utilizado como base o método $k$-means para definir um método intervalar para segmentação de imagens digitais, denominado de método $k$-means intervalar.

\section{Segmentação de Imagens por Agrupamento}

O método de agrupamento é um processo segundo o qual um conjunto de dados é separado em vários grupos (clusters) [4] e os elementos de cada grupo possuem similaridades entre si, mas são dissimilares entre os elementos de outros grupos. Um dos métodos para segmentação de imagens pode ser tratado através de métodos de agrupamentos, por exemplo o $k$-means, onde, a imagem é separada em partes ou objetos significativos, e cada parte constitui em um conjunto de pixels que foram agrupados de acordo com alguma similaridade, por exemplo, a textura, o nível de cinza, e/ou a vizinhança, entre outras medidas de similaridades entre os pixels.

O interesse na segmentação de imagens por agrupamento é formar grupos (segmentos) onde, os elementos (os pixels) de cada grupo possuem maior similaridade entre si e menor similaridade entre os elementos de outros grupos. A métrica mais utilizada é a Euclidiana [5], mas também é conhecido outras métricas, tais como, D4 ou Distância Quarteirão [5], ou também D8 ou Distância Xadrez [5].

O método $k$-means para segmentação de imagens particiona a imagem original 
em $k$ grupos e atribui a cada grupo um valor médio que representará a intensidade luminosa (ou nível de cinza). Este método busca diminuir a quantidade de níveis de cinza da imagem original para $k$ quantidades, e a média de valores dos elementos (ou pixels) de cada grupo representa uma intensidade luminosa, logo, a imagem terá somente $k$ intensidades luminosas diferentes divididos em $k$ agrupamentos para uma posterior análise dessa imagem segmentada.

Diversos algoritmos podem representar o método $k$-means, o algoritmo que segue é uma dessas representações para o método $k$-means para segmentação de imagens.

\section{Algoritmo de segmentação de imagens por $k$-means}

- passo 1: entre com a imagem, e inicializar: a quantidade $k$ de grupos (clusters), os valores iniciais dos $k$ centróides e definir o critério de parada;

- passo 2: calcular a similaridade entre os pixels com cada centróide;

- passo 3: atribuir cada pixel com o grupo do centróide mais similar;

- passo 4: calcular a média de cada grupo e atribuir esse valor a cada centróide de acordo com os grupos;

- passo 5: verificar o critério de parada, caso não seja satisfeito, voltar ao passo 2.

O método de segmentação por $k$-means particiona a imagem original em $k$ grupos, onde, cada grupo ou parte possui um valor médio (um centróide) diferente que corresponde à representação de níveis de cinza da imagem, considerando imagens monocromáticas. Para o cálculo da medida de similaridade pode ser utilizado a distância Euclidiana entre o centróide e o pixel, e esta medida definirá em qual grupo esse pixel pertencerá. Um dos critérios de parada considerado é a não variação dos pixels entre os grupos, ou seja, se o valor dos centróides não variarem, comparando os centróides atuais com os anteriores durante a execução do método, então o método encontrou um resultado que convergiu.

A divisão de uma imagem em várias partes ou agrupamentos faz com que haja uma perda de informações na imagem segmentada. Várias pesquisas na área de processamento de imagens estão voltadas para o desenvolvimento de métodos, inclusive o de segmentação, que retornem resultados com o mínimo de perda de informações possível. Os métodos intervalares para segmentação de imagens fazem com que haja um maior controle de erros computacionais, sendo uma proposta para métodos de segmentação de imagens de alta precisão.

\section{Imagens Digitais Intervalares}

A definição de imagem digital refere-se a uma representação de uma função bidimensional de intensidade luminosa $f(x, y)$ em que o valor de amplitude $f$ nas coordenadas espaciais $(x, y)$ dá a intensidade da imagem no ponto $(x, y)[5]$. A definição de imagem digital intervalar [9] refere-se a uma função luminosa bidimensional $F(x, y)$, 
onde, um intervalo assume a amplitude $F$ nas coordenadas espaciais $(x, y)$ dando a intensidade da imagem no ponto $(x, y)$. Dessa forma, a representação de uma imagem em formato digital intervalar, ou seja, uma imagem digital intervalar, possivelmente possuirá uma descrição mais concisa de imagem original para a imagem digital.

Uma imagem digital intervalar, ou brevemente, imagem intervalar, pode ser representado por uma matriz intervalar $A=a_{i j}$ de ordem $m \times n$. Os elementos da matriz $A$, os $a_{i j}$ 's (os pixels), representam as intensidades luminosas intervalares em cada posição $i, j$ da matriz $A$.

Na Figura 1 o item (a) representa uma imagem digital intervalar e o item (b) representa os pixels intervalares da parte selecionada na imagem intervalar.
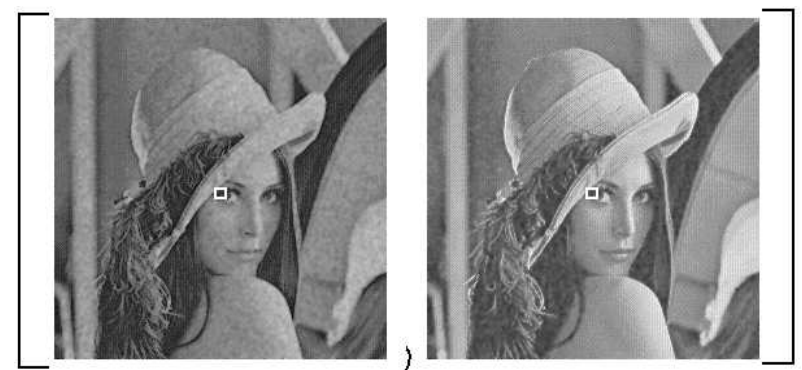

(a)

\begin{tabular}{|l|l|l|l|l|l|}
\hline$[147,170]$ & {$[131,153]$} & {$[137,153]$} & {$[146,187]$} & {$[155,187]$} & {$[161,187]$} \\
\hline$[144,187]$ & {$[130,153]$} & {$[138,170]$} & {$[145,187]$} & {$[152,204]$} & {$[163,187]$} \\
\hline$[147,153]$ & {$[138,153]$} & {$[145,170]$} & {$[156,204]$} & {$[163,187]$} & {$[163,187]$} \\
\hline$[145,153]$ & {$[144,153]$} & {$[149,170]$} & {$[158,204]$} & {$[153,204]$} & {$[147,204]$} \\
\hline$[141,153]$ & {$[155,187]$} & {$[149,204]$} & {$[158,187]$} & {$[139,187]$} & {$[102,125]$} \\
\hline$[154,187]$ & {$[162,187]$} & {$[162,204]$} & {$[163,204]$} & {$[102,120]$} & {$[102,120]$} \\
\hline
\end{tabular}

(b)

Figura 1: Representação de uma Imagem Digital Intervalar.

Como ainda não existe nenhum dispositivo capaz de gerar uma imagem intervalar, existem algumas formas de manipular a formação de imagens intervalares, entre uma dessas formas é gerar uma imagem intervalar através de várias outras imagens de uma mesma cena, onde o intervalo será definido através do menor e do maior valor dos pixels em cada posição $(x, y)$ nas várias imagens utilizadas. Dessa forma, a construção da imagem intervalar será mais sensível às informações, tendo em vista que terá uma quantidade maior de informações (através de várias imagens de uma mesma cena) para a formação de uma imagem.

O processamento digital de imagens intervalares é uma teoria nova, e ainda não possui um sistema de processamento de imagens digitais intervalares que trate totalmente por intervalos. O que atualmente existe são algumas técnicas que tratam de problemas encontrados principalmente na aquisição da imagem utilizando conceitos da matemática intervalar, e também técnicas para a etapa de pré-processamento para melhorar a imagem eliminando ruídos e/ou realçando a imagem [9]. 
O sistema de processamento de imagens digitais pode ser dividido em: aquisição da imagem, definição do problema, pré-processamento, segmentação, representação e descrição, e análise. Para o sistema de processamento de imagens digitais intervalares pode ser seguido a mesma estrutura do sistema de processamento de imagens digitais, desde que seja tratado por imagens intervalares e respeitando as teorias intervalares. Em pré-processamento, como foi mencionado anteriormente, já encontra-se estudos realizados para imagens intervalares, fazendo uso da transformada de Fourier $[9,10]$. Neste estudo, será mostrado um método intervalar para o processo de segmentação considerando a sua importância na fase de análise.

\section{Método de Segmentação Intervalar}

Nesta sessão, busca-se definir um método para realizar a etapa de segmentação de imagens digitais na versão intervalar, que tem como objetivo buscar resultados seguros, ou seja, resultados que tenham controle de erros computacionais.

A segmentação de imagens intervalares, ou brevemente, segmentação intervalar, consiste em subdividir uma imagem intervalar em partes ou objetos significativos. Este processo deve ser tratado com considerável atenção, pois, otimiza a análise da imagem intervalar e possivelmente a identificação de um melhor resultado. A segmentação intervalar pode ser abordado de várias formas, dentre elas, em métodos totalmente intervalares, onde, busca-se um maior controle de erros computacionais, uma vez que é tratado desde o início por imagens intervalares e por métodos intervalares; e em métodos parcialmente intervalares, ou seja, métodos intervalares que utilizam imagens digitais reais, e que têm como objetivo minimizar erros computacionais (arredondamento e/ou truncamento) somente durante o processamento do método. Este estudo mostrará somente o método totalmente intervalar para segmentação de imagens digitais, denominado-o de método intervalar.

O método intervalar para segmentação utilizado foi baseado no método $k$-means, denominado de $k$-means intervalar, sendo uma extensão do método $k$-means, e que tem como objetivo buscar resultados mais confiáveis durante o processo de segmentação.

O método k-means intervalar, para segmentação, particiona uma imagem intervalar em $k$ grupos de acordo com a similaridade do centróide intervalar dos grupos com os pixels intervalares da imagem. A similaridade entre os pixels intervalares e os centróides intervalares é determinado através de uma métrica intervalar, a função de distância entre o pixel e o centróide. As métricas intervalares utilizadas são, a métrica usual da matemática intervalar, a métrica de Moore [12, 13] e a Quasi-métrica [1].

Sejam $P=\left[p_{1}, p_{2}\right]$ e $Q=\left[q_{1}, q_{2}\right]$ dois valores intervalares, então as métricas intervalares são:

- Métrica de Moore: $D_{M}=\max \left(\left|p_{1}-q_{1}\right|,\left|p_{2}-q_{2}\right|\right)$;

- Quasi-métrica: $D_{Q M}=\max \left(q_{1}-p_{1}, p_{2}-q_{2}, 0\right)$.

$\mathrm{O}$ algoritmo do método de segmentação por $k$-means intervalar pode ser descrito da seguinte forma, 


\section{Algoritmo do método $k$-means intervalar}

- passo 1: entre com a imagem intervalar, e inicializar: a quantidade de $k$ agrupamentos, os valores intervalares iniciais dos $k$ centróides intervalares, e definir o critério de parada;

- passo 2: calcular a similaridade entre os pixels intervalares com cada centróide intervalar;

- passo 3: atribuir cada pixel intervalar com o grupo de centróide intervalar mais similar;

- passo 4: calcular a média de cada grupo e atribuir esse valor intervalar a cada centróide intervalar;

- passo 5: verificar o critério de parada, caso não satisfaça o critério de parada, voltar ao passo2.

O critério de parada utilizado no $k$-means intervalar foi a estabilização dos valores intervalares dos centróides, igual ao critério de parada do $k$-means, e uma quantidade máxima de épocas, ou seja, uma época é caracterizado pelo novo valor dos centróides intervalares encontrados durante a execução do método intervalar.

$\mathrm{O}$ método $k$-means intervalar para segmentação agrupa os pixels nos grupos de acordo com a similaridade dos centróides, por exemplo, um certo pixel possui o valor intervalar de [75, 80], se houver dois centróides cujos valores são: [80, 82] e [90,95], então, o pixel $[75,80]$ será mais similar do centróide [80,82] de acordo com as duas métricas intervalares.

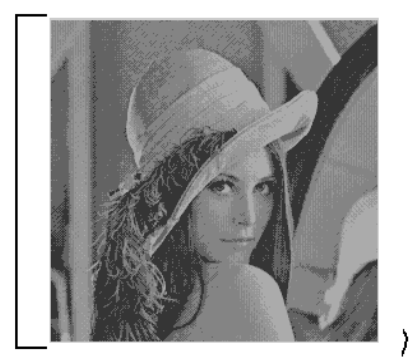

(a)

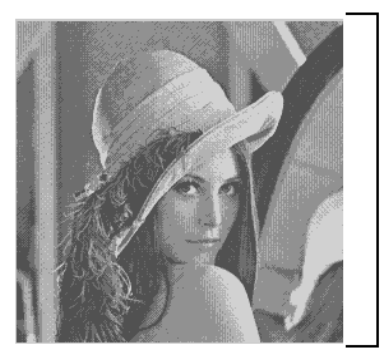

(b)

Figura 2: Resultado da aplicação do método de segmentação intervalar por k-means.

A segmentação pelo método $k$-means intervalar tem como resultado uma imagem intervalar, cujos pixels foram agrupados de acordo com a similaridade da intensidade de cada pixel, visando um método que controle possíveis erros computacionais através da teoria intervalar, e que destaca possíveis regiões de nebulosidade, ou seja, se um pixel possuir similaridade igual em mais de um centróide, este pixel será classificado em ambos os grupos pertencentes aos centróides, com isso, essa região deverá ter uma análise mais detalhada devido a essa nebulosidade. 
A Figura 2 mostra uma imagem intervalar segmentada em oito grupos, ou seja, $k=8$, através do método $k$-means intervalar. Os itens (a) e (b) representam os ínfimos e os supremos, respectivamente, da imagem intervalar segmentada.

\section{Resultados e Conclusões}

O método intervalar para segmentação de imagens, o $k$-means intervalar, foi aplicado em um conjunto de trinta imagens de $64 \times 64$ pixels de 256 níveis de cinza, sendo dividido em três classes: imagens de animais, imagens de frutas e imagens de personagens animados.

Uma análise comparativa foi feita entre as métricas intervalares, distância de Moore e distância Quasi-métrica, com o método k-means intervalar, e foi feita uma análise comparativa entre o método $k$-means intervalar e o usual. Foram usados valores diferentes para $k(k=2, k=4$ e $k=8)$, isto é, para dois, quatro e oito grupos, respectivamente.

Essas análises comparativas foram feitas através do erro médio da segmentação de cada imagem, gerando uma média desses erros para todas as trinta imagens. $\mathrm{O}$ erro médio é a diferença entre a imagem original e a imagem segmentada, e ele pode ser considerado um discriminante para uma classificação (ou segmentação) boa ou ruim, de acordo com o valor do erro.

Tabela 1: Erro Médio Total - Distância Euclidiana e Distância de Moore.

\begin{tabular}{|c|c|c|c|}
\hline \multicolumn{4}{|c|}{ Erro Médio Total } \\
\hline \multicolumn{4}{|c|}{ k-means - distância Euclidiana } \\
\hline & $\mathrm{k}=\mathbf{2}$ & $\mathrm{k}=4$ & $\mathrm{k}=\mathbf{8}$ \\
\hline Erro Médio & 27,37202 & 12,90037 & 6,76889 \\
\hline Desvio Padrão & 4,68776 & 1,57266 & 0,63127 \\
\hline \multicolumn{4}{|c|}{ k-means intervalar - distância de Moore } \\
\hline & $\mathrm{k}=\mathbf{2}$ & $\mathrm{k}=4$ & $\mathrm{k}=\mathbf{8}$ \\
\hline Erro Médio & 24,70725 & 16,28257 & 10,40431 \\
\hline Desvio Padrão & 5,90387 & 2,65924 & 1,64083 \\
\hline \multicolumn{4}{|c|}{ k-means intervalar - distância Quasi-métricc } \\
\hline & $\mathrm{k}=\mathbf{2}$ & $\mathrm{k}=4$ & $\mathrm{k}=\mathbf{8}$ \\
\hline Erro Médio & 23,43337 & 12,37929 & 6,65143 \\
\hline Desvio Padrão & 7,09686 & 2,09492 & 0,99856 \\
\hline
\end{tabular}

A Tabela 1 refere-se ao erro médio total, ou seja, o erro médio de todas as imagens analisadas, de cada métrica (real ou intervalar), e dos métodos: $k$-means e $k$-means intervalar.

O erro médio total do método $k$-means, na Tabela 1 , mostra-se maior que o método $k$-means intervalar, tanto para a métrica de Moore, quanto para a Quasimétrica, podendo considerar uma melhor segmentação através do método $k$-means 
intervalar. A análise comparativa entre as métricas intervalares, houve um ganho com a métrica Quasi-métrica, como visto na Tabela 1.

A diminuição dos erros médios totais quando aumenta a quantidade de grupos, para $k=2, k=4$ e $k=8$, mostra que, quanto maior a quantidade de grupos menor o erro, ou seja, a segmentação por agrupamento terá erro menor. A escolha do valor de $k$ agrupamentos é dependente do problema a ser resolvido.

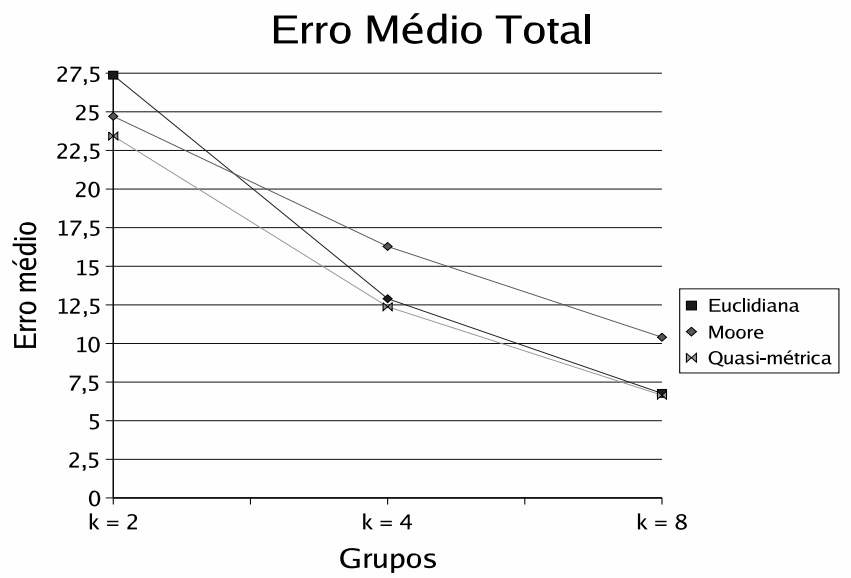

Figura 3: Erro médio total.

A Figura 3 compara os erros médios totais entre o método de segmentação intervalar por $k$-means intervalar e o método usual. No método intervalar temos também uma comparação com as métricas de Moore e a Quasi-métrica.

A Tabela 2 refere-se à quantidade média de épocas que o método (real ou intervalar) demorou para convergir.

O comparativo do método $k$-means com o $k$-means intervalar, para as métricas, Euclidiana e Moore, através da Tabela 2, mostra que para $k=2$, e $k=4$, o método $k$-means foi mais rápido, porém, quando aumentou para $k=8$ houve uma ligeira perda. Já a comparação do método $k$-means com o $k$-means intervalar, com as métricas, Eucliciana e Quasi-métrica, o método $k$-means foi mais rápido para convergir. Sendo que, a métrica intervalar Quasi-métrica perde para as outras duas métricas (real e intervalar). 
Tabela 2: Quantidade de Épocas - Distância Euclidiana e Distância de Moore.

\begin{tabular}{|c|c|c|c|}
\hline \multicolumn{4}{|c|}{ Quantidade de Épocas } \\
\hline \multicolumn{4}{|c|}{ k-means - distância Euclidiana } \\
\hline & $\mathrm{k}=\mathbf{2}$ & $\mathrm{k}=4$ & $\mathrm{k}=8$ \\
\hline Épocas & 7,93333 & 20,33333 & 38,73333 \\
\hline Desvio Padrão & 1,84270 & 6,18780 & 12,06906 \\
\hline \multicolumn{4}{|c|}{ k-means intervalar - distância de Moore } \\
\hline & $\mathrm{k}=\mathbf{2}$ & $\mathrm{k}=4$ & $\mathrm{k}=\mathbf{8}$ \\
\hline Épocas & 9,13333 & 22,86666 & 38,33333 \\
\hline Desvio Padrão & 2,62974 & 9,33714 & 24,69728 \\
\hline \multicolumn{4}{|c|}{ k-means intervalar - distância Quasi-métricc } \\
\hline & $\mathrm{k}=\mathbf{2}$ & $\mathrm{k}=4$ & $\mathrm{k}=\mathbf{8}$ \\
\hline Épocas & 9,93333 & 24,63333 & 47,43333 \\
\hline Desvio Padrão & 4,08193 & 11,51081 & 24,18771 \\
\hline
\end{tabular}

\section{Referências}

[1] B.R.C. Bedregal e B.M. Acióly, A Quasi-metric Topology Compatible with Inclusion Monotonicity on Interval Space, International Journal Realiable Computing, 3 (1997), 305-310.

[2] G.B. Coleman e H.C. Andrew, Image Segmentation by Clustering, em "Procedings of IEEE", pp. 773-785, 1979.

[3] V. Faber, Clustering and the Continuous K-means Algorithm, em "los Alamos Science", Vol. 22, pp. 138-144,, 1994.

[4] D.A. Forsyth e J Ponce, "Computer Vision: A Modern Approach", Prentice Hall, 2002.

[5] R.C. Gonzalez e R.E. Woods, "Processamento de Imagens Digitais", Edgard Blucher Ltda, 2000.

[6] T. Kanungo, D.M. Mount, N.S. Netanyahu, R. Silverman, C. Piatko e A.Y. $\mathrm{Wu}$, The Analysis of a Simple K-means Clustering Algorithm, em "Proceeding of the Sixteent Annual Symposium on Computational Geometry", pp. 100-200, 2000 .

[7] V. Kreinovich, A. Lakeyev, J. Rohn e P. Kahl, "Computational Complexity and Feasibility of Data Processing and Interval Computations", Kluwer Academic Publishers, 1998.

[8] S.P. Lloyd, Least Square Quantization in PCM, IEEE Transactions on Information Theory, 28 (1982), 129-137. 
[9] A. Lyra, "Uma Fundamentação Matemática para o Processamento de Imagens Intervalares", PhD Thesis, LECA, Universidade Federal do Rio Grande do Norte, 2003.

[10] A. Lyra, B.R.C. Bedregal, R.C. Bedregal e A.D.D. Neto, The Interval Images Processing, em "WSEAS Transactions on Circuits and Systems", pp. 229-233, 2004.

[11] R.E. Moore, Automatic Error Analysis in Digital Computation. "Tech. Report LMSD 84821 Lockheed Missiles and Space Co.", 1959.

[12] R.E. Moore, "Interval Arithmetic and Automatic Error Analysis in Digital Computing", PhD Thesis, Technical Report n. 25, 1962.

[13] R.E. Moore, "Interval Analysis". Prentice Halls, 1966. 